Ann. Zootech., I975, 24 (I), 8I-94.

\title{
INFLUENCE DE LA STRUCTURE PHYSIQUE ET DE LA QUALITÉ DE CONSERVATION DES ENSILAGES DE GRAMINÉES SUR LEUR INGESTIBILITÉ
}

\author{
J.-P. DULPHY, G. BECHET et E. THOMSON \\ avec la collaboration technique de J.-M. Borssau, H. Bousouet, \\ Jacqueline Jamot, Marie Jailler et L. L'Hotelier \\ Laboratoive des Aliments, \\ Centre de Recherches de Clermont-Ferrand, I. N. R. A., \\ Theix, Saint Genès Champanelle, 63110 Beaumont
}

\section{RÉSUMF́,}

I. Nous avons effectué un essai sur des moutons pour tenter d'expliquer l'influence de la qualité de conservation et de la finesse de hachage sur les quantités d'ensilage ingérées. Pour cela nous avons offert à volonté à des moutons trois ensilages de brome différant par la longueur de leurs brins et 3 ensilages de fléole de même longueur de brins, mais de qualité de conservation différente. Parallèlement aux mesures de digestibilité et d'ingestibilité nous avons mesuré :

- la vitesse de digestion des ensilages dans le rumen;

- l'importance des contenus de rumen ;

- les manifestations du comportement alimentaire et mérycique des moutons.

2. L'ingestibilité des ensilages utilisés a été d'autant plus élevée que leur hachage, avant ou après la mise en silo, était plus fin et que leur qualité de conservation était meilleure.

3. L'activité cellulolytique du jus de rumen n'a pas différé significativement d'un ensilage à l'autre. De même la cinétique de digestion des ensilages n'a pas été affectée par la qualité de conservation ou la longueur des brins. Les différences d'ingestibilité entre ensilages issus de la même plante ne peuvent donc pas être cxpliquées par des variations de leur vitesse de digestion dans le rumen.

4. Les poids de contenu frais ou sec du réticulo-rumen ont été d'autant plus importants que l'ingestibilité des ensilages a été plus élevée. On a noté ccpendant un allongement considérable du temps de séjour dans le rumen pour l'ensilage à brins longs. La distension du rumen ne limite clonc pas les quantités ingérées pour les ensilages les moins bien consommés.

5. Lorsque les moutons ont ingéré des brins longs nous avons noté également des difficultés importantes de rumination, un faible nombre de petits repas au cours de la journée et la présence de longues particules dans le rumen.

Il semble donc que la cause principale de la faible ingestibilité chez le mouton des ensilages à brins longs soit la difficulté de les régurgiter pour les ruminer. Il suffit de hacher ces ensilages à brins longs pour qu'une rumination normale se rétablisse et que le nombre de repas et par là la quantité journalière ingérée augmente.

6. En revanche, il est beaucoup plus difficile d'interpréter l'influence de la qualité de conservation. Cette dernière joue essentiellement sur la vitesse d'ingestion et sur la quantité ingérée 
lors du repas principal suivant les distributions d'ensilage. Le fait-elle par l'intermédiaire du goût, de l'odeur, agit-elle directement sur l'appétit, ou sur certains aspects de la digestion? Il est difficile d'opter pour l'une ou l'autre de ces hypothèses.

\section{INTRODUCTION}

Les variations de l'ingestibilité des ensilages chez le mouton sont liées en grande partie à leur finesse de hachage et, pour une même longueur de brins, à leurs caractéristiques fermentaires (DULPHY et DEMARQUILLY, I972 et I973). On connaît cependant très mal les causes exactes de ces liaisons. D'après l'étude du comportement alimentaire des animaux, il semble que l'odeur et le goût interviennent peu (DulPhy, I972a) et n'expliquent pas la faible ingestibilité de certains ensilages. D'autre part, il est possible que dans les ensilages, l'augmentation de la teneur en acides et en formes dégradées de l'azote (ammoniac et amines) ait une action inhibitrice sur l'appétit des ruminants (Moore, Thomas et Sykes, rg6o; McLeod, Wilikins et Raymond, I970; Demarquili, , I973). Cependant, cette action inhibitrice ne peut être que partielle car il suffit de hacher des ensilages à brins longs à la sortie du silo pour augmenter leur ingestibilité (MURDOCH, I965; DULPHY et Demarquiliy, I972). Nous poursuivons donc des études pour savoir si les quantités ingérées d'ensilage ne sont pas en partie soumises à une régulation de type "physique ", c'est-à-dire une régulation dans laquelle interviennent en particulier la vitesse de digestion du fourrage dans le rumen, la vitesse de transfert des particules indigestibles du rumen vers les compartiments digestifs postérieurs et le volume des cavités gastriques.

En effet, dans un premier essai (DulPHy, I972b), nous avions observé que les quantités ingérées et les vitesses de digestion de fourrages verts, fanés ou ensilés (vitesse de digestion mesurées sur des échantillons secs et broyés placés dans des sachets suspendus dans le rumen) variaient dans le même sens. Dans cet essai, la faible ingestibilité du seul ensilage à brins longs étudié ne pouvait cependant pas être expliquée par sa vitesse de digestion. Cela nous conduit à mener de nouveaux essais dans lesquels nous avons étudié l'influence de la qualité de conservation et de la finesse de hachage de différents ensilages sur :

- la vitesse de leur digestion dans le rumen appréciée par la technique des sachets de nylon;

- l'importance des contenus de rumen (+ réseau) correspondant (en poids ou en volume) ;

- les manifestations du comportement alimentaire et mérycique des animaux.

\section{MATÉRIEL E'T MÉTHODES}

\section{Fourrages}

Nous avons utilisé :

- 4 fourrages de brome dont 3 ensilages récoltés à la même date dans la même prairie, mais avec 3 machines de récolte différentes donnant des brins courts (I à $2 \mathrm{~cm}$ ), moyens (5 à 
INGESTIBILITÉ DES ENSILAGES DE GRAMINÉES

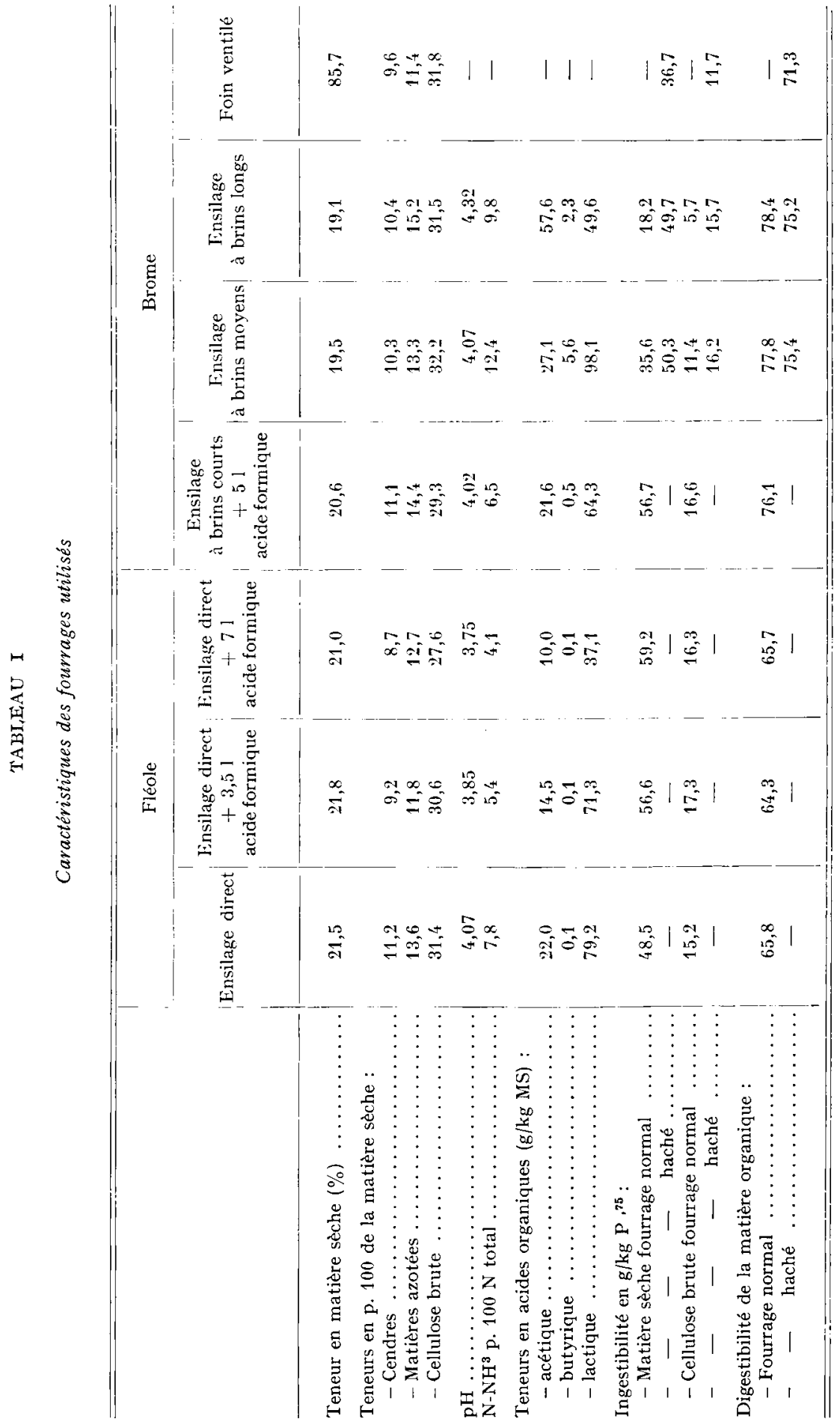




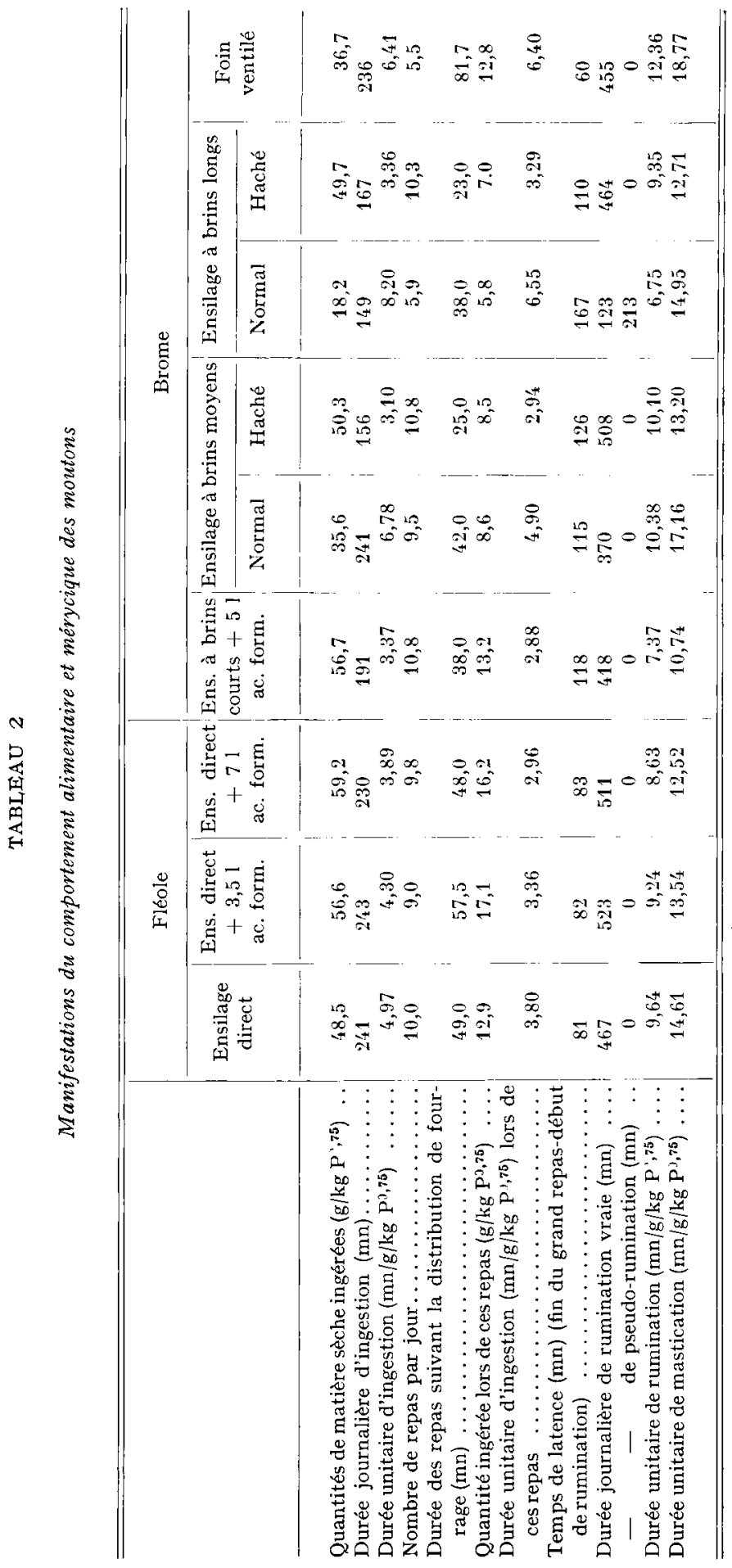


ro $\mathrm{cm}$ ) ou longs (ro à $20 \mathrm{~cm}$ ) et un foin provenant de la même parcelle, mais fauché une semaine plus tard.

- 3 ensilages de fléole récoltés à la même date dans la même prairie avec une même machine de récolte (brins courts de I à $2 \mathrm{~cm}$ ), mais conservés avec addition de $0,3,5$ et 7 litres d'acide formique à $80 \mathrm{p}$. Ioo par tonne de fourrage frais.

Les caractéristiques de ces fourrages sont rapportées dans le tableau $\mathbf{I}$.

\section{Animaux}

Nous avons travaillé sur moutons (mâles castrés de race Texel) âgés de 2 à 3 ans, pesant 50 à $60 \mathrm{~kg}$ et recevant les fourrages ad libitum (Io p. Ioo de refus par jour) en deux distributions par jour à $8 \mathrm{~h}$ et $\mathbf{i} 6 \mathrm{~h}$. Ces moutons étaient placés en cage à métabolisme et disposaient en permanence d'eau et de sel (pierre à lécher).

\section{Mesures}

La digestibilité et l'ingestibilité de ces 7 fourrages, mesurées sur Io moutons par les méthodes précédemment décrites (DULPHy et DEMARQUILLY, I972 et I973), sont reportées dans le tableau I. Les manifestations du comportement alimentaire ont été étudiées à partir de l'enregistrement des mouvements de mâchoire dans le même temps et reportées dans le tableau 2. Pour l'ensilage à brins moyens et celui à brins longs de brome, les moutons ont été séparés en 2 lots de 5, l'un recevant l'ensilage tel quel et l'autre l'ensilage haché dans un hache paille (brins d'environ 2 à $3 \mathrm{~cm}$ ).

Les 7 fourrages ont été distribués à volonté au cours de périodes successives de 3 semaines à 2 moutons porteurs d'une fistule de rumen; les ensilages hachés au hache paille n'ont pas été distribués à ces moutons fistulés. Ces derniers étaient adaptés à leur régime pendant 9 jours puis les diverses mesures avaient lieu pendant les iz jours suivants : quantités ingérées durant les repas suivant les 2 distributions journalières à 8 et $\mathbf{I} 6 \mathrm{~h}$, quantités ingérées par jour, cinétique de digestion des fourrages dans le rumen et quantité de contenu sec et frais du rumen.

La cinétique de la digestion du fourrage dans le rumen a été mesurée par la méthode des sachets de nylon (Demarouilly et Chenost, r969). Des sachets, contenant le même fourrage ( $3 \mathrm{~g}$ de matière sèche par sachet) que celui ingéré par les moutons, ont été placés durant I 3, 24 et 48 heures dans le rumen de chacun des 2 moutons fistulés en même temps que les sachets contenant un fourrage témoin (paille d'avoine broyée) pour apprécier l'activité cellulolytique du jus de rumen. Chaque mesure a été effectuée en double et répétée à une semaine d'intervalle.

De plus, pour les 4 fourrages de brome, les digestions en sachets ont, non seulement été mesurées avec du fourrage sec et broyé, mais aussi avec du fourrage mastiqué (prélevé durant l'ingestion sur un mouton muni d'une fistule de l'œsophage) et légèrement séché à l'étuve jusqu'à 30-40 p. Ioo de matière sèche.

La digestion en sachets des 3 ensilages de brome séchés et broyés a également été mesurée en utilisant une vache recevant un fourrage vert de ray-grass.

Quarante vidages du contenu du réticulo-rumen des moutons fistulés ont été effectués à la main après ouverture de la canule du rumen. Ils ont été effectués juste avant et $2 \mathrm{~h}$ après la distribution du repas du matin et du soir, chaque vidage étant séparé du suivant d'au moins 48 heures. Ils ont au lieu 36 à $4^{8}$ heures avant la pose ou après la sortie des sachets de nylon de manière à ne pas perturber l'étude de la vitesse de digestion. Après vidage, le contenu était pesé, échantillonné pour mesurer la teneur en matière sèche et replacé très rapidement dans le rumen.

\section{RÉSULTATS}

\section{Quantités ingérées et comportement alimentaire des moutons non fistulés (tabl. 2)}

L'amélioration de la qualité de conservation des ensilages de fléole, entraînée par l'addition d'une dose croissante d'acide formique (o, 3,5 et 71 /tonne) a et1 un effet très net sur leurs quantités ingérées par jour et par repas principal et a dimi- 
nué nettement leur durée unitaire d'ingestion (DUI ; voir définition dans DULPHY, I97r). Elle a de même diminué la durée unitaire de rumination (DUR) et de mastication (DUM) (ingestion + rumination).

La quantité ingérée des ensilages de brome a augmenté très nettement avec leur finesse de hachage. Il reste cependant une différence d'ingestibilité entre l'ensilage haché avant la mise en silo et ceux hachés après, due à une différence de qualité de conservation et peut-être aussi à une légère différence de longueur de brins (à la sortie du hache paille, les brins d'ensilage mesurent en effet 2 à $3 \mathrm{~cm}$, contre I à $2 \mathrm{~cm}$ pour les ensilages hachés avant la mise en silo). Les animaux ingérant l'ensilage à brins longs ingèrent très lentement (DUI élevée) et font peu de repas. Ils ruminent également pendant moins de temps mais la rumination correspondante semble très efficace (DUR faible) du moins si on ne considère que la rumination vraie; mais il faut tenir compte également de l'importance de la rumination à vide (rumination sans remontée de bols alimentaires se traduisant par des cycles incomplets de rumination sur les bandes d'enregistrement) par rapport à la durée de rumination vraie. Cette rumination à vide est très importante avec l'ensilage à brins longs puisqu'elle représente 2 I3 minutes en 24 heures contre I23 minutes pour la rumination vraie. D'autre part, pour l'ensilage à brins longs la première période de rumination (après la fin des grands repas) commence avec beaucoup de retard (50 minutes environ) sur celle correspondant aux ensilages à brins courts.

Notons enfin que les quantités de cellulose brute ingérées sont beaucoup moins différentes que les quantités de matière sèche ingérées (tabl. r) : en moyenne $\mathrm{I} 6,3 \mathrm{~g} / \mathrm{kg} \mathrm{P}^{\mathbf{0}, 75}$ pour les ensilages de fléole et $\mathrm{I} 6,2 \mathrm{~g} / \mathrm{kg} \mathrm{P}^{0,75}$ pour les ensilages de brome hachés finement.

\section{Digestibilité des fourrages in vivo (tabl. I)}

Les ensilages issus de la même plante sur pied ont eu des digestibilités pratiquement égales entre eux car identiques à celle du fourrage vert de départ. Ira qualité de conservation ou la finesse de hachage n'ont donc pas eu d'influence sur la digestibilité des ensilages. Certes les ensilages de brome à brins moyens et longs ont eu des digestibilités légèrement stupérietures (respectivement de I,7 et 2,3 points) à celles de l'ensilage à brins courts mais cela tient uniquement à leur faible niveau d'ingestion. Il a, en effet, suffi de les hacher au hache paille pour que leur digestibilité diminue par suite de l'augmentation de leur niveau d'ingestion.

En revanche, le foin de brome a été nettement moins digestible que les ensilages de brome mais il avait été fauché une semaine plus tard.

\section{Digestibilité des fourrages en sachets (tabl. 3-4-5)}

L'activité cellulolytique du jus de rumen mesurée par la digestibilité de la paille en sachets ne diffère pas significativement d'un ensilage à un autre ensilage correspondant à la même plante. On ne trouve pas non plus de différences significatives entre ensilages, foin et herbe verte, ainsi qu'entre la fléole et le brome, ou entre la vache et les moutons.

La cinétique de digestion des ensilages n'est pas significativement affectée par la qualité de conservation ou la longueur des brins. Dans le cas des ensilages 
de brome mastiqués on note cependant une digestibilité 13 heures en sachets de l'ensilage à brins courts un peu plus faible que celle des ensilages à brins moyens ou longs. La différence n'est pas significative et ne se retrouve pas à 24 et 48 heures. Elle doit résulter d'une diminution aléatoire et passagère de l'activité cellulolytique du jus de rumen puisque la digestibilité I3 heures de la paille (effectuée en même temps) a, elle aussi été un peu plus faible qu'avec les autres régimes.

\section{TABLEAU 3}

Activité cellulolytique (jugée par la digestion en sachets de nylon de la paille) du jus de rumen des moutons recevant les différents ensilages de fléole et cinétique de digestion de ces différents ensilages

\begin{tabular}{|c|c|c|c|}
\hline & Ensilage direct & $\begin{array}{l}\text { Ensilage direct } \\
+3,5 \text { litres } \\
\text { acide formique }\end{array}$ & $\begin{array}{l}\text { Ensilage direct } \\
+7 \text { litres } \\
\text { acide formique }\end{array}$ \\
\hline $\begin{array}{c}\text { Quantités ingérées } \\
\text { par les moutons fistulés } \\
\text { (g de matière sèche/kg } \mathbf{P}^{0}, 7 \bar{b} \text { ) }\end{array}$ & 50,2 & 56,7 & 67,8 \\
\hline 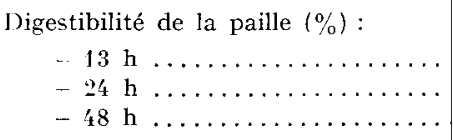 & $\begin{array}{l}32,2 \\
46,3 \\
65,4\end{array}$ & $\begin{array}{l}28,5 \\
43,3 \\
63,9\end{array}$ & $\begin{array}{l}30,6 \\
46,9 \\
67,2\end{array}$ \\
\hline 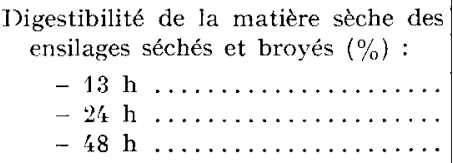 & $\begin{array}{l}57,0 \\
68,5 \\
79,7\end{array}$ & $\begin{array}{l}53,7 \\
67,1 \\
78,2\end{array}$ & $\begin{array}{l}56,2 \\
70,2 \\
80,8\end{array}$ \\
\hline $\begin{array}{l}\text { Digestibilité de la matière sèche inso- } \\
\text { luble dans l'eau (MS-extrait aqueux) } \\
\text { réellement digérée par les micro- } \\
\text { organismes du rumen }(\%) \text { : } \\
\quad-13 \mathrm{~h} \ldots \ldots \ldots \ldots \ldots \ldots \ldots \ldots \\
\quad-24 \mathrm{~h} \ldots \ldots \ldots \ldots \ldots \ldots \ldots \ldots \\
-48 \mathrm{~h}\end{array}$ & $\begin{array}{l}43,0 \\
58,2 \\
73,0\end{array}$ & $\begin{array}{l}38,0 \\
55,9 \\
70,8\end{array}$ & $\begin{array}{l}41,5 \\
60,2 \\
74,5\end{array}$ \\
\hline
\end{tabular}

Contenus de rumen

Les quantités ingérées par les moutons fistulés ne sont pas toujours identiques à celles des moutons intacts placés en cage à métabolisme mais elles varient dans le même sens (tabl. 3 et 4 ). L'importance du contenu de leur rumen ( + réseau) peut donc nous renseigner en partie sur l'importance du contenu de rumen des moutons intacts. Les poids de matière fraîche ou de matière sèche, ainsi que le volume du contenu, varient toujours de la même manière au cours de la journée quels que soient les fourrages ingérés : ils sont minimum avant le repas du matin, augmentent de façon importante lors du grand repas du matin, diminuent très légèrement dans la journée et sont maximum après le repas du soir (fig. I). 


\section{TABLEAU 4}

Activité cellulolytique du jus de rumen des moutons recevant les différents ensilages de brome et cinétique de digestion de ces différents ensilages

\begin{tabular}{|c|c|c|c|c|}
\hline & $\begin{array}{c}\text { Ensilage } \\
\text { à brins courts }\end{array}$ & $\begin{array}{c}\text { Ensilage } \\
\text { à brins moyens }\end{array}$ & $\begin{array}{c}\text { Ensilage } \\
\text { à brins longs }\end{array}$ & Foin \\
\hline $\begin{array}{c}\text { Quantités ingérées } \\
\text { par les moutons fistulés } \\
\left.\text { (g MS/kg } \mathrm{P}^{0,75}\right)\end{array}$ & 53,4 & 28,2 & 12,3 & 45,7 \\
\hline 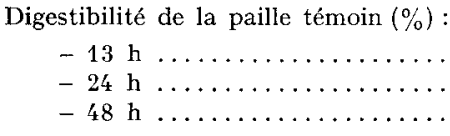 & $\begin{array}{l}32,1 \\
48,1 \\
68,9\end{array}$ & $\begin{array}{l}36,4 \\
53,2 \\
69,2\end{array}$ & $\begin{array}{l}36,4 \\
56,1 \\
71,0\end{array}$ & $\begin{array}{l}30,9 \\
47,7 \\
67,6\end{array}$ \\
\hline 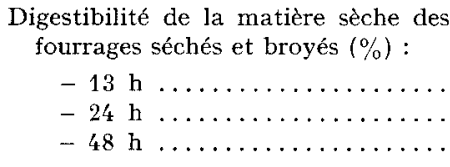 & $\begin{array}{l}61,1 \\
76,1 \\
87,6\end{array}$ & $\begin{array}{l}62,8 \\
75,0 \\
85,7\end{array}$ & $\begin{array}{l}64,2 \\
80,9 \\
88,6\end{array}$ & $\begin{array}{l}56,3 \\
71,2 \\
83,1\end{array}$ \\
\hline 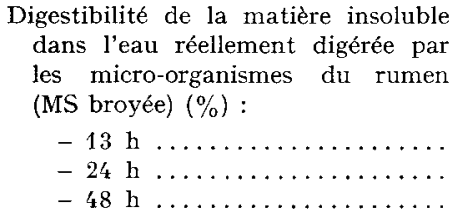 & $\begin{array}{l}44,5 \\
65,9 \\
81,6\end{array}$ & $\begin{array}{l}45,1 \\
63,1 \\
78,9\end{array}$ & $\begin{array}{l}48,3 \\
72,4 \\
83,5\end{array}$ & - \\
\hline 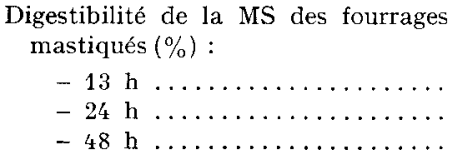 & $\begin{array}{l}64,2 \\
77,6 \\
86,5\end{array}$ & $\begin{array}{l}68,8 \\
79,5 \\
86,9\end{array}$ & $\begin{array}{l}70,0 \\
79,8 \\
87,2\end{array}$ & $\begin{array}{l}56,1 \\
69,0 \\
82,4\end{array}$ \\
\hline $\begin{array}{l}\text { Digestibilité de la MS insoluble dans } \\
\text { l'eau réellement digérée par les } \\
\text { micro-organismes (MS mastiquée) : } \\
\quad-13 \mathrm{~h} \ldots \ldots \ldots \ldots \ldots \ldots \ldots \ldots \ldots \ldots \\
-24 \mathrm{~h} \ldots \ldots \ldots \ldots \ldots \ldots \ldots \ldots \ldots\end{array}$ & $\begin{array}{l}49,0 \\
67,0 \\
79,7\end{array}$ & $\begin{array}{l}54,0 \\
69,8 \\
80,4\end{array}$ & $\begin{array}{l}56,6 \\
70,8 \\
81,5\end{array}$ & $\begin{array}{l}-- \\
- \\
-\end{array}$ \\
\hline
\end{tabular}




\section{TABLEAU 5}

Cinétique de digestion des ensilages de brome dans le rumen d'une vache

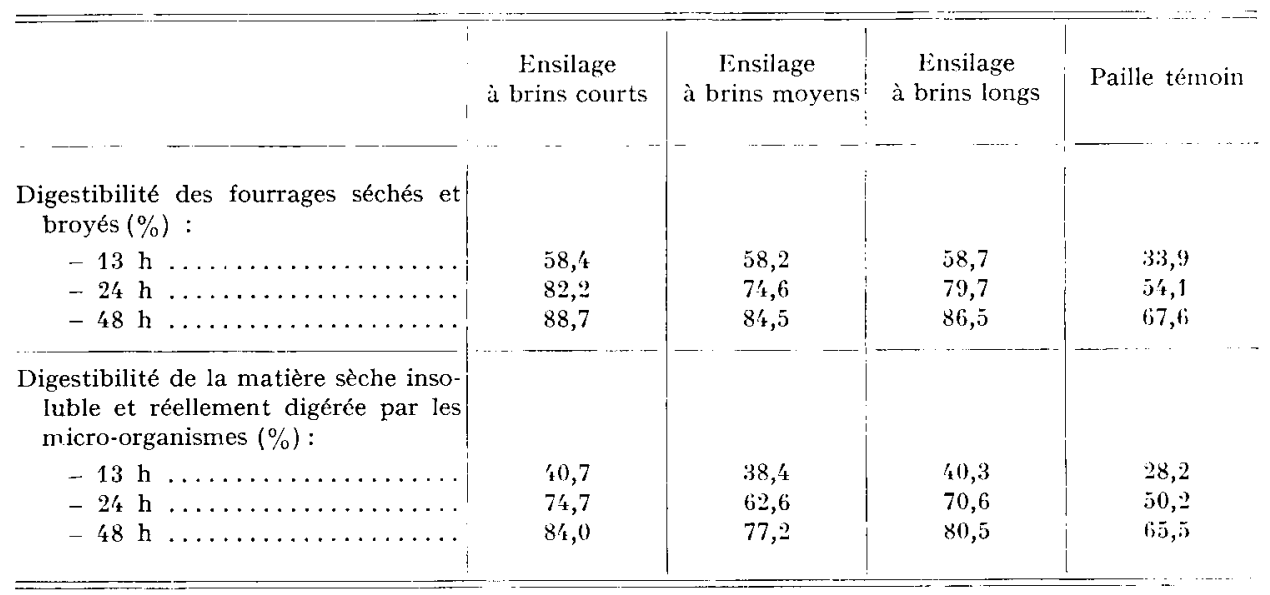

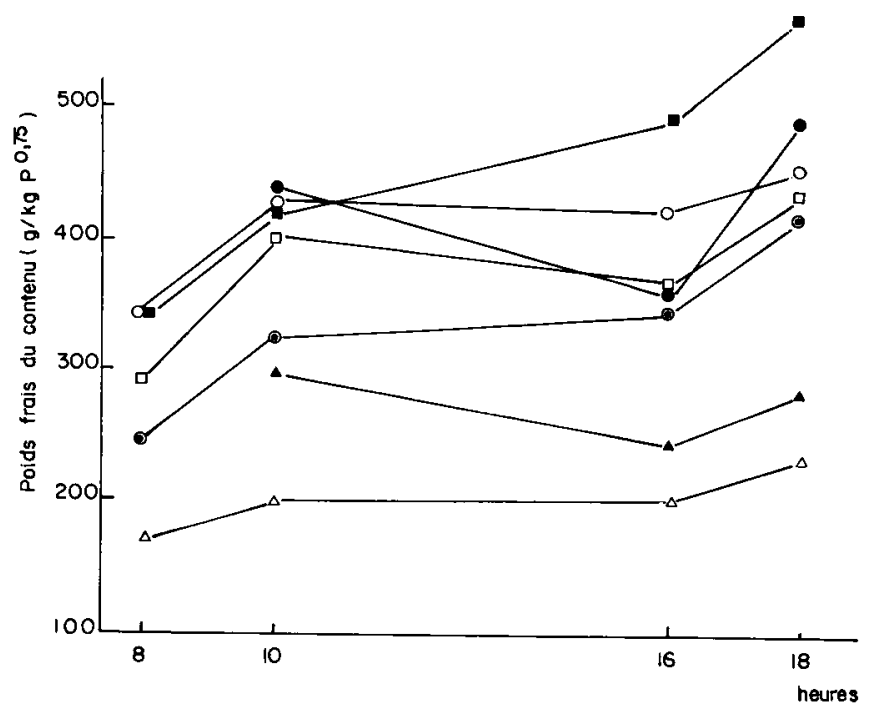

FI G. I. - Évolution du poids frais du contenu de rumen au cours de la journée

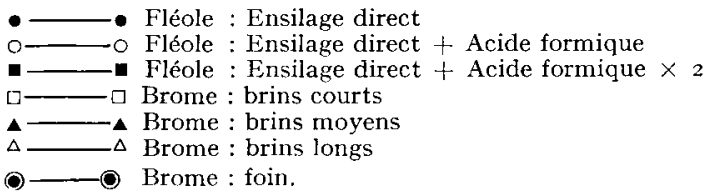


Les teneurs en matière sèche des contenus augmentent légèrement au cours des repas, de 9,44 à IO, I 9 le matin et de Io,07 à I I,43 le soir en regroupant 1'ensemble des contenus. Les deux ensilages à brins moyens et longs se distinguent cependant car ils conduisent à des contenus très aqueux (teneurs en matière sèche comprise entre 7 et 9 p. IOo). De plus, lorsque les moutons ingèrent l'ensilage à brins longs on retrouve systématiquement dans le rumen un amas important de longues particules, ce qui rejoint les observations de Murdoch (I965). Enfin, pour l'ensemble des fourrages, la densité moyenne des contenus diminue faiblement de 933 à $905 \mathrm{~g} /$ litre au cours du repas du matin et de 932 à $920 \mathrm{~g} /$ litre au cours du repas du soir.

Pour comparer l'importance ou les caractéristiques des contenus suivant les différents régimes nous avons donc calculé une moyenne pondérée prenant en compte les 4 mesures effectuées par régime (tabl. 6). Dans ces conditions, il apparaît

\section{TABLEAU 6}

Influence du régime sur l'importance du contenu de rumen et ses caractéristiques

\begin{tabular}{|c|c|c|c|c|c|c|c|}
\hline \multirow{2}{*}{$=\cdots=-\cdots$} & \multicolumn{3}{|c|}{ Tléole } & \multicolumn{4}{|c|}{ Brome } \\
\hline & $\begin{array}{l}\text { Ensilage } \\
\text { direct }\end{array}$ & $\begin{array}{c}\text { Ensilage } \\
\text { direct } \\
+ \text { ac. } \\
\text { form. }\end{array}$ & $\begin{array}{c}\text { Ensilage } \\
\text { direct } \\
+ \text { ac. } \\
\text { form. } \times 2\end{array}$ & $\begin{array}{c}\text { Ensilage } \\
\text { brins } \\
\text { courts }\end{array}$ & $\begin{array}{l}\text { Ensilage } \\
\text { brins } \\
\text { moyens }\end{array}$ & $\begin{array}{c}\text { Ensilage } \\
\text { brins } \\
\text { longs }\end{array}$ & Foin \\
\hline $\begin{array}{ccc}-\cdots & - & -\end{array}$ & $\ldots-$ & & & & $\ldots$ & $\ldots$ & $--\cdots$ \\
\hline Poids des moutons $(\mathrm{k} y)$ & 51 & 51 & 51 & 51 & 51 & 51 & 51 \\
\hline $\begin{array}{l}\text { Quantités ingérées de ma- } \\
\text { tiere seche (g) par jour }\end{array}$ & 1020 & 1275 & 1365 & 1050 & 750 & 200 & 922 \\
\hline $\begin{array}{c}\text { Quantités ingérées par } \\
\text { grands repas }(\mathrm{g}) \ldots \ldots\end{array}$ & 306 & 306 & 470 & 270 & 170 & 80 & 300 \\
\hline Poids de contenu frais $(\mathrm{g})$. & 7800 & 8050 & 9150 & 7080 & 5400 & 3950 & 6570 \\
\hline Poids de contenu $\sec (\mathrm{g})$ & 873 & 890 & 1176 & 740 & 453 & 303 & 632 \\
\hline $\begin{array}{c}\text { Teneur en matière sèche } \\
(\%) \ldots \ldots \ldots \ldots \ldots \ldots\end{array}$ & 11,20 & 11,03 & 12,85 & 10,45 & 8,40 & 7,70 & 9,65 \\
\hline Volume du contenu (ml). & 8375 & - & - & 7850 & 5870 & 4015 & 7220 \\
\hline Densité du contenu $(\mathrm{g} / \mathrm{l})$. & 932 & $\longrightarrow$ & 一 & 902 & 921 & 952 & 910 \\
\hline
\end{tabular}

que les poids du contenu frais ou sec du rumen sont d'autant plus importants que 1'ingestibilité des ensilages est plus élevée. Ils augmentent avec la qualité de conservation des ensilages de fléole, et avec la finesse de hachage des ensilages de brome. Ils sont plus importants pour les ensilages que pour le foin dans la mestre où les ensilages sont ingérés en plus grande quantité que le foin :

Le rapport $\frac{\text { quantité journalière moyenne de contenu sec du rumen }}{\text { quantité journalière moyenne de matière sèche ingérée }} \times 24$ nous donne une idée de séjour moyen (en heures) dans le rumen. Les valeurs obtenues qui sont respectivement pour les ensilages (o, 3,5 et 71 d'acide formique/tonne) de fléole, les ensilages de brome brins courts, moyens et longs et le foin de brome, 
2I,I ; I7,0 ; 20,$9 ; \mathrm{I}_{7}, 3$; I5,I ; 38,4 et I6,3 heures, mettent clairement en évidence un allongement considérable du temps de séjour dans le rumen pour l'ensilage de brome à brins longs.

\section{DISCUSSION}

La vitesse de digestion intrinsèque des fourrages dépend de deux paramètres (DulPHy, I972 b) : d'une part l'activité microbienne du jus de rumen, d'autre part, la résistance des fourrages à la digestion microbienne. Dans nos essais, ces deux paramètres n'ont pas été modifiés de façon significative par la qualité de conservation des ensilages distribués ni par la longueur de leurs brins. Il est d'ailleurs intéressant de constater que l'activité cellulolytique du jus de rumen mesurée par la digestibilité en sachets à I3, 24 ou 48 heures de la paille a toujours été comparable quel que soit le régime (les différents ensilages ou le foin) reçu par les moutons, de même qu'elle a été comparable chez le mouton recevant ces différents fourrages conservés et chez la vache ingérant un fourrage vert. L'activité microbienne du jus de rumen des moutons ingérant des ensilages est donc tout à fait normale. D'autre part, il n'y a pas eu de différences entre les résistances à la digestion microbienne de la matière sèche des ensilages issus du même fourrage sur pied. En outre, la comparaison des résultats obtenus dans les sachets avec les fourrages simplement mastiqués et ceux broyés montrent que la différence de présentation des fourrages agit peu sur la cinétique et l'intensité de leur digestion. Dans nos conditions, les différences de quantités ingérées entre ensilages issus de la même plante sur pied ne peuvent donc pas être expliquées par des variations de leur vitesse de digestion intrinsèque dans le rumen.

On constate cependant pour les ensilages à brins longs un allongement considérable du temps de séjour dans le rumen apprécié par le rapport de la quantité de contenu du rumen à la quantité journalière ingérée. C'est qu'en effet le temps de séjour d'un fourrage dans le rumen dépend à la fois de sa vitesse de digestion (la digestion des tissus cellulosiques (parenchyme) étant indispensable à la libération des tissus lignifiés indigestibles (schlerenchyme, vaisseaux...)) et de la vitesse de réduction de ces tissus indigestibles en particules suffisamment fines pour franchir l'orifice du feuillet (JARrige, Demarguiliy et Dulphy, I973). Or, cette vitesse de réduction en fines particules dépend dans une large mesure de l'efficacité de la rumination. Quand le mouton ingère des ensilages à brins longs nous avons observé une perturbation importante de son comportement mérycique. L'animal a des difficultés à ruminer : le temps de latence entre la fin du repas et le début de la rumination est long et quand la rumination démarre, elle se caractérise par de nombreux pseudo cycles, c'est-à-dire des cycles de rumination ne correspondant qu'à la remontée de jus. La rumination ne devient normale que lorsque le fourrage a déjà subi une digestion microbienne assez longue ; elle ne devient par exemple normale que vers 6 heures du matin, après le repas distribué à 16 heures la veille. La présence de très longues fibres enchevêtrées dans le rumen après le repas, déjà signalée par CAMPLING (I968) doit gêner considérablement la régurgitation des bols alimentaires et cette régurgitation ne devient possible que lorsque les fibres ont été plus ou moins 
scindées en particules plus courtes par la digestion microbienne et par le brassage du contenu du rumen. Il semble que le mouton avale les brins longs d'ensilage sans les scinder tous (les études en cours au laboratoire sur des moutons munis d'une fistule de l'œsophage le confirment) et qu'il lui soit ensuite difficile de les régurgiter. Il en résulte un allongement du temps de séjour dans le rumen, mais qui ne peut cependant expliquer la faible quantité ingérée puisqu'en même temps le rumen est beaucoup moins plein que lorsque le Mouton reçoit des ensilages à brins courts ou $\mathrm{du}$ foin. Les résultats que nous avons obtenus, confirmant les observations de Thomas et al. (I96I), de Waldo et al. (I965) et de Campling (Ig66) montrent en effet que ce n'est pas la distention du rumen (+ réseau) qui limite les quantités d'ensilages ingérées du moins de ceux dont la qualité de conservation est faible ou dont la longueur des brins est grande, puisque le volume ou le poids du contenu varie dans le même sens que les quantités ingérées. Il semble donc que la cause principale de la faible ingestibilité chez le mouton des ensilages à brins longs soit la difficulté de les régurgiter pour les ruminer. Les moutons ayant des difficultés à ruminer, font peu de repas et mangent donc peu, bien que leur rumen soit peu rempli. Il suffit que ces ensilages à brins longs soient hachés avant leur distribution aux animaux pour qu'une rumination normale se rétablisse et que le nombre de repas et par là la quantité journalière ingérée augmentent.

L'influence de la qualité de conservation des ensilages à brins courts sur leur ingestibilité est, par contre, beaucoup plus difficile à interpréter. Nous constatons en effet que la qualité de la conservation joue essentiellement sur la vitesse d'ingestion et sur la quantité ingérée lors du repas principal suivant les distributions d'ensilage (fig. 2). Certes l'influence de la qualité de conservation sur la vitesse

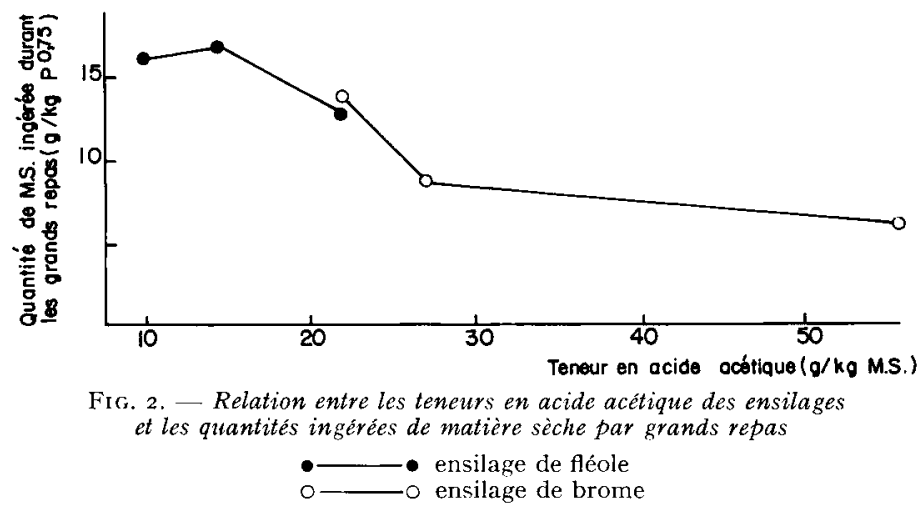

d'ingestion pourrait s'exercer par l'intermédiaire du goût ou de l'odeur mais pourquoi donc les animaux ne consomment-ils pas pendant plus longtemps pour ingérer autant au repas principal comme nous l'observons pour les ensilages longs distribués tels quels ou après hachage. La qualité de conservation agit-elle donc directement sur l'appétit par l'intermédiaire de certains métabolites apportés par l'ensilage comme l'ont envisagé MoOre (I960), McLEOd et al. (I970), Demarquilly (I973). Il est difficile d'opter pour l'une ou l'autre des hypothèses d'autant plus qu'il est curieux de constater que le temps mis à ruminer rapporté à la quantité de cellulose 
brute ingérée est le même pour les trois ensilages de fléole $\left(30,2\right.$ à $\left.3 \mathrm{I}, 4 \mathrm{mn} / \mathrm{g} / \mathrm{kg} \mathrm{P}^{\mathbf{0}, 75}\right)$. L'augmentation relative de la teneur en membranes des ensilages mal conservés ne peut-elle donc pas aussi expliquer en partie leur ingestibilité un peu plus faible bien qu'avec ces ensilages la quantité de contenu du rumen n'ait pas encore atteint son maximum, et qu'il n'y ait pas de différence dans la cinétique de digestion en sachets de ces ensilages.

En définitive, la régulation des quantités ingérées d'ensilage pourrait être, pour une part, qu'il reste à définir, soumise à la fois à des facteurs d'ordre physique (JARRige, DEMARquiliy et DULPHY, I973) et à la fois des facteurs d'ordre métabolique. Nous continuerons à envisager ces deux hypothèses en développant également des études sur bovins, ces derniers étant beaucoup moins sensibles que les ovins à la longueur des brins d'ensilage (MURDOCh, I965; Dulphy et DEMARQuilly, I973).

Recu pour publication en juillet 1974 .

\section{SUMMARY}

\section{INFLUENCE OF THE PHYSICAL STRUCTURE AND QUALITY OF CONSERVATION OF GRASS SILAGES ON THEIR VOLUNTARY INTAKE BY SHEEP}

I. A trial was made on sheep with the aim of explaining the influence of the conservation quality and chopping fineness on the silage intake. For this purpose, sheep were fed ad libitum with 3 brome-grass silages of different silages of different chopping finenesses and with 3 timothy silages of the same chopping fineness but of different conservation qualities (table I). Parallel to the measurements of the digestibility and voluntary intake, the following parameters were determined :

- digestion rate of the silages in the rumen,

- weight and volume of rumen contents,

- feeding and ruminating behaviour of sheep.

2. The voluntary intake of the silages was all the higher as the chopping of the silage before or after ensiling was finer and as the conservation quality of the silages was better, $i$. $e$. with lower ammonia and volatile fatty acid contents (table I).

3. The following observations were made when the sheep were fed the coarsely chopped silage : - low ingestion rate and small number of meals per day;

- rumination difficulty characterized by the fact that the first rumination period after the end of the main meals was much delayed and by numerous periods of in vacuo rumination (without any ascending of the alimentary bolus) (table 2 ).

4. The cellulotic activity of the rumen fluid did not differ from one silage to another and was absolutely comparable with that of the animals receiving green forage or hay. Likewise, the digestion kinetics of the silages made from the same original herbage were not affected by the quality of conservation or the chopping fineness of the silage (tables 3,4 and 5 ). Thus, the differences in the voluntary intake of silages made with the same original herbage cannot be explained by variations in their digestion rate in the rumen.

5. The weight of the fresh or dry reticulo-ruminal contents before or after the morning or evening meal differed much from one silage to another; they were all the higher as the voluntary intake of the silages was more important (fig. I, table 6). However, a considerable lengthening of the time of staying in the rumen was noticed for the coarsely chopped silage ( 38.4 hours versus I 5. I to 2 I.I hours for the other silages) as well as the presence of long particles in the rumen.

Thus, the distension of the rumen did not limit the amount ingested of the silages with the lowest intake levels.

6. It therefore seems that the main causc of the low voluntary intake of coarsely chopped silages by the sheep is the regurgitation difficulty before rumination. A further chopping of the 
silages is enough to re-establish a normal rumination and to increase the number of meals and consequently the daily feed intake.

7. On the other hand, it is much more difficult to explain the influence of the quality of conservation. The latter mainly affect the ingestion rate and the amount of food eaten during the main meal following the administration of silage (fig. 2). Does this influence depend on the taste or the odour or does it act directly on the appetite or on some aspects of the digestion? It is difficult to decide which of these hypotheses is the most valid one.

\section{RÉFÉRENCES BIBLIOGRA PHIQUES}

Campling R. C., I966. The intake of hay and silage by cows. J. Brit. Grassland Soc., 21, 4I-48.

Demarguilly C., Chenost M., ig69. Étude de la digestion des fourrages dans le rumen par la méthode des sachets de nylon. Liaison avec la valeur alimentaire des fourrages. Ann. Zootech., 18, 4I9-436.

Demarquilly C., I973. Composition chimique, caractéristiques fermentaires, digestibilité et quantité ingérée des ensilages de fourrages : modifications par rapport au fourrage vert initial. Ann. Zootech., 22, I-35.

Dulphy J.-P., I97I. Influence du poids vif et du niveau d'ingestion sur le comportement alimentaire et mérycique du Mouton. Ann. Zootech., 20, 477-486.

Dulphy J.-P., I972 a. Étude de quelques relations entre le mode de conservation du fourrage ingéré et le comportement alimentaire et mérycique des moutons. Ann. Zootech., 21, 429-44I.

Dulphy J.-P., I972 b. Influence du mode de conservation des fourrages de graminées sur la vitesse de leur digestion dans le rumen. Ann. Zootech., 21, 525-534.

Dulphy J.-P., Demarquilly C., I972. Influence de la machine de récolte sur Ia valeur alimentaire des ensilages. Ann. Zootech., 21, I63+173.

Dulphy J.-P., Demargully $\mathrm{C}$, I973. Influence de la machine de récolte et de la finesse de hachage sur la valetrr alimentaire des ensilages. Ann. Zootech., 22, 199-217.

Jarrige R., Demarovilly C., Dulphy J.-P., I973. The voluntary intake of forages. 12th Eupop. Grassland Congress Uppsala.

More L. A., Thomas J. W., Sykes J. F., ig6o. The acceptability of grass legume silage by dairy cattle. Proc. 8th Int. Grassl. Congr. Reading 7or-704.

McLeod D. S., Wilkins R. J., Raymond W. F., I970. The voluntary intake by sheep and cattle of silages differing in free-acid content. J. Agric. Sci. Camb., 75, 31 I-319.

Murdoch J. C., I965. The effect of length of silage on its voluntary by cattle. J. Brit. Grassl. Soc., 20, $54-58$.

Thomas J. W., Moore L. A., Sykes J. K., I96r. Further comparisons of alfalfa hay and alfalfa silage for growing dairy heifers. J. dairy Sci., 44, 862-873.

Waldo D. R., Miller R. W., Oкamoto M., Moore L. A., i965. Ruminant utilization of silage in relation to hay, pellets, and hay plus grain : II. Rumen content, Dry matter passage and water intake. J. Dairy Sci., 48, I I, I473. 